\title{
The large-scale magnetic field of Proxima Centauri near activity maximum
}

\author{
Baptiste Klein ${ }^{1}{ }^{\star}{ }^{\text {Jean-François Donati }}{ }^{1}$, Élodie M. Hébrard ${ }^{1}$, Bonnie Zaire ${ }^{1}$, \\ Colin P. Folsom ${ }^{1}$, Julien Morin ${ }^{2}$, Xavier Delfosse ${ }^{3}$, Xavier Bonfils $^{3}$ \\ ${ }^{1}$ Université de Toulouse, CNRS, IRAP, 14 av. Belin, 31400 Toulouse, France \\ ${ }^{2}$ LUPM, Université de Montpellier, CNRS, Place Eugène Bataillon, F-34095 Montpellier, France \\ ${ }^{3}$ CNRS, IPAG, Université Grenoble Alpes, 38000 Grenoble, France
}

Accepted. Received in original form 2020 October 27

\begin{abstract}
We report the detection of a large-scale magnetic field at the surface of the slowly-rotating fully-convective M dwarf Proxima Centauri. Ten circular polarization spectra, collected from April to July 2017 with the HARPS-Pol spectropolarimeter, exhibit rotationally-modulated Zeeman signatures suggesting a stellar rotation period of $89.8 \pm 4.0 \mathrm{~d}$. Using Zeeman-Doppler Imaging, we invert the circular polarization spectra into a surface distribution of the largescale magnetic field. We find that Proxima Cen hosts a large-scale magnetic field of typical strength $200 \mathrm{G}$, whose topology is mainly poloidal, and moderately axisymmetric, featuring, in particular, a dipole component of $135 \mathrm{G}$ tilted at $51^{\circ}$ to the rotation axis. The large-scale magnetic flux is roughly $3 \times$ smaller than the flux measured from the Zeeman broadening of unpolarized lines, which suggests that the underlying dynamo is efficient at generating a magnetic field at the largest spatial scales. Our observations occur $\sim 1 \mathrm{yr}$ after the maximum of the reported 7 yr-activity cycle of Proxima Cen, which opens the door for the first long-term study of how the large-scale field evolves with the magnetic cycle in a fully-convective verylow-mass star. Finally, we find that Proxima Cen's habitable zone planet, Proxima-b, is likely orbiting outside the Alfvèn surface, where no direct magnetic star-planet interactions occur.
\end{abstract}

Key words: techniques: polarimetric - stars: low-mass - stars: magnetic field - stars: rotation - stars: individual: Proxima Centauri

\section{INTRODUCTION}

Late $\mathrm{M}$ dwarfs are primary targets in the quest for Earth twins (Kasting et al. 1993). Their small masses and radii, as well as close-in habitable zones (HZ), make the detection of temperate Earth-like planets around them easier than around solar-like stars. As a result, the most favorable planets to further investigate habitability with forthcoming telescopes like the JWST and ELTs orbit stars with spectral type later than M4 (e.g., Berta-Thompson et al. 2015; Anglada-Escudé et al. 2016; Gillon et al. 2017; Dittmann et al. 2017; Astudillo-Defru et al. 2017b). However, these stars exhibit strong magnetic activity (e.g., West et al. 2011), whose manifestations, such as high-energy winds or frequent flaring events, are likely to affect the properties of the planets in their HZ. Therefore, understanding the processes underlying activity phenomena is a major prerequisite to study the conditions of habitability around low-mass stars (Lammer et al. 2007; France et al. 2016).

Late M dwarfs are fully-convective (FC; Baraffe et al. 1998).

^ E-mail: baptiste.klein@irap.omp.eu
Their underlying dynamo processes remain mysterious, despite recent advances in the explanation of observations by numerical models (Yadav et al. 2015, 2016). Spectropolarimetric observations of FC stars have revealed a bimodal distribution of their magnetic properties with either strong axisymmetric dipoles, or weaker nonaxisymmetric complex fields (Donati et al. 2006a; Morin et al. 2008, 2010; Kochukhov \& Lavail 2017). The origin of this bimodality, tentatively explained by bistability in the dynamo process (Morin et al. 2011; Gastine et al. 2012, 2013), or a single oscillatory dynamo process (Kitchatinov et al. 2014), is still debated in the literature. However, most of the observational results currently available involve very active stars whose dynamo lies in the so-called saturated regime, i.e., on the plateau of the activity-Rossby number ${ }^{1}$ relationship (typically, Rossby number $R o \lesssim 0.1$ which corresponds to a rotation period of roughly $10 \mathrm{~d}$ for a mid-M dwarf; Pizzolato et al. 2003; Kiraga \& Stepien 2007; Shulyak et al. 2017; Astudillo-Defru

\footnotetext{
1 The Rossby number is defined as the stellar rotation period normalized to the convective turnover time, set to $\sim 143_{-22}^{+31} \mathrm{~d}$ for Proxima Cen using Wright et al. (2018) empirical relationship.
} 


\section{$2 \quad$ B. Klein et al.}

et al. 2017a; Wright et al. 2018; See et al. 2019). Hence the interest in observing the magnetic field of FC M dwarfs in the unsaturated dynamo regime like Proxima Centauri (rotation period of $\sim 90 \mathrm{~d}$ ), whose large-scale magnetic properties are still unconstrained.

Spectropolarimetry is the best way to model the large-scale topologies of stellar magnetic fields, and thereby the underlying dynamo processes powering magnetic activity. At the photospheric level, magnetic fields produce circularly-polarized Zeeman signatures that can be inverted into a map of the large-scale magnetic field using Zeeman-Doppler imaging (ZDI, Donati \& Landstreet 2009). Spectropolarimetric monitoring of FC M dwarfs is thus expected to bring new constraints on the nature of the dynamo processes operating in their interiors. Moreover, the large-scale magnetic geometry is an essential ingredient for modelling stellar winds, known to play a major role in star-planet interactions (SPI, Vidotto et al. 2014; Strugarek et al. 2015).

Our closest neighbor, Proxima Centauri, is an active FC M5.5 dwarf whose dynamo lies in the unsaturated regime ( $R o=0.63, \log L_{\mathrm{X}} / L_{\mathrm{bol}}=-3.94$; Wright et al. 2018). Proxima Cen hosts a HZ Earth-mass planet, Proxima-b (Anglada-Escudé et al. 2016), whose habitability conditions have been widely studied in the literature (e.g., Ribas et al. 2016; Turbet et al. 2016; Barnes et al. 2016; Meadows et al. 2018). This planet orbits at less than 0.05 au from its host star, and its atmospheric and surface properties are likely affected by stellar magnetic activity (e.g., Garraffo et al. 2016; Garcia-Sage et al. 2017). Moreover, the star exhibits a 7 yrphotometric activity cycle (Suárez Mascareño et al. 2016; Wargelin et al. 2017), interpreted as resulting from a $\alpha \Omega$-dynamo at work in its convective interior (Yadav et al. 2016). Spectropolarimetric observations of Proxima Cen can guide theoretical dynamo models and provide key inputs to investigate SPIs with the close-in planet.

In this study, we present the first reconstruction of Proxima Cen's large-scale magnetic topology from a set of 10 spectropolarimetric observations collected with HARPS-Pol from April to July 2017, around the activity maximum. In Sec. 2, we present our spectropolarimetric observations and detection of magnetic field. We then detail, in Sec. 3, the modelling of the Zeeman signatures and their inversion into the large-scale magnetic topology of Proxima Cen. Finally, we discuss the implications of our results regarding the activity and magnetic cycle of the star, and its extended magnetosphere, in Sec. 4.

\section{OBSERVATIONS}

Spectropolarimetric observations of Proxima Cen were collected using the spectropolarimetric mode of the High Accuracy Radial velocity Planet Searcher velocimeter (HARPS-POL; Mayor et al. 2003; Snik et al. 2011, resolving power: $\sim 115000$, wavelength domain: 378-691 nm). From April to July 2017, 10 sequences of circular polarisation spectra were obtained (ESO program 099.C0334(A), PI: Hébrard), each sequence consisting of four individual spectra taken in different configurations of the retarder. The data reduction is carried out using ESPaDOnS's nominal data reduction pipeline Libre-ESpRIT (e.g., Donati et al. 2006b), inspired by the reduction process described in Donati et al. (1997), and adapted to HARPS-Pol spectra (see Hébrard et al. 2016). The four subexposures within each spectropolarimeteric sequence are combined to extract Stokes $I$ (unpolarized) and Stokes $V$ (circularly-polarized) spectra in a way to remove systematics and correct for spurious polarization signatures to the first order in circular polarization spectra, (Donati et al. 1997). The peak signal-to-noise ratios (S/N) per pixel
Table 1. List of HARPS-Pol spectropolarimetrc observations of Proxima Cen (ESO program 099.C-0334(A), PI: Hébrard). All polarization sequences consist of 4 individual subexposures of $1200 \mathrm{~s}$ each. Columns 1 to 3 indicate the observation dates, BJDs (at mid-exposure), and peaks of S/N (per $0.85 \mathrm{~km} \mathrm{~s}^{-1}$ velocity bin), respectively. Columns 4 and 5 list the rms noise level relative to the unpolarized continuum level per pixel bin in each Stokes $V$ spectrum and the estimated longitudinal magnetic field $\mathrm{B}_{\ell}$ (with $1 \sigma$ error bars). The stellar rotation phase, computed from the reference time $\mathrm{BJD}=2457862$ and a rotation period of $89.8 \mathrm{~d}$, is given in column 6 .

\begin{tabular}{cccccc}
\hline Date & $\begin{array}{c}\text { BJD } \\
{[2457000+]}\end{array}$ & $\mathrm{S} / \mathrm{N}$ & $\begin{array}{c}\sigma_{\mathrm{V}} \\
{\left[10^{-4} I_{\mathrm{C}}\right]}\end{array}$ & $\begin{array}{c}\mathrm{B}_{\ell} \\
{[\mathrm{G}]}\end{array}$ & Phase \\
\hline $2017 / 04 / 18$ & 862.7525 & 130 & 4.5 & $3.2 \pm 8.2$ & 0.008 \\
$2017 / 04 / 28$ & 872.7921 & 154 & 3.8 & $-27.7 \pm 6.6$ & 0.120 \\
$2017 / 05 / 04$ & 878.7772 & 157 & 3.7 & $-73.5 \pm 6.9$ & 0.187 \\
$2017 / 05 / 13$ & 887.8047 & 78 & 7.0 & $-36.7 \pm 18.8$ & 0.287 \\
$2017 / 05 / 15$ & 889.8118 & 91 & 6.4 & $-52.0 \pm 15.1$ & 0.310 \\
$2017 / 06 / 18$ & 923.6802 & 105 & 5.4 & $5.8 \pm 12.5$ & 0.687 \\
$2017 / 06 / 29$ & 934.5817 & 96 & 5.7 & $12.7 \pm 14.9$ & 0.808 \\
$2017 / 07 / 02$ & 937.6045 & 145 & 4.0 & $5.2 \pm 8.0$ & 0.842 \\
$2017 / 07 / 09$ & 944.6375 & 165 & 3.5 & $19.4 \pm 6.7$ & 0.920 \\
$2017 / 07 / 14$ & 949.5841 & 106 & 5.0 & $-10.6 \pm 2.5$ & 0.975 \\
\hline
\end{tabular}

Table 2. Stellar parameters of Proxima Cen used in this study.

\begin{tabular}{ccc}
\hline Parameter & Value & Reference \\
\hline Spectral type & M5.5 & Bessell (1991) \\
Distance & $1.3012 \pm 0.0003 \mathrm{pc}$ & Gaia Collaboration et al. (2018) \\
$T_{\text {eff }}$ & $2980 \pm 80 \mathrm{~K}$ & Ribas et al. (2017) \\
$\log g$ & $5.02 \pm 0.18$ & Passegger et al. (2016) \\
$M_{\mathrm{S}}$ & $0.120 \pm 0.003 \mathrm{M}_{\odot}$ & Ribas et al. (2017) \\
$R_{\mathrm{S}}$ & $0.146 \pm 0.007 \mathrm{R}_{\odot}$ & Ribas et al. (2017) \\
$<B_{\mathrm{I}}>$ & $600 \pm 150 G(3 \sigma)$ & Reiners \& Basri (2008) \\
$i$ & $47 \pm 7^{\circ}$ & This work \\
$P_{\text {rot }}$ & $89.8 \pm 4.0 \mathrm{~d}$ & This work \\
$v \sin i$ & $0.06 \pm 0.01 \mathrm{~km} \mathrm{~s}^{-1}$ & This work \\
$R o$ & $0.63_{-0.10}^{+0.14}$ & Wright et al. (2018) \\
\hline
\end{tabular}

$\left(0.85 \mathrm{~km} \mathrm{~s}^{-1}\right.$ velocity bin) of the extracted spectra range from 78 to 165 , with a median value of 118 . The full journal of observations is given in Table 1.

We apply Least-Squares Deconvolution (LSD, Donati et al. 1997 ) to compute average Stokes $I$ and Stokes $V$ profiles for our 10 spectra. This is done using a mask of atomic lines computed from an ATLAs9 local thermodynamical equilibrium model assuming an effective temperature of $3000 \mathrm{~K}$ and a surface gravity of 5.0 (Kurucz 1993). The final mask contains $\sim 4000$ moderate-to-strong lines (i.e., with depth larger than $40 \%$ as advocated in Donati et al. 1997) covering the entire HARPS domain. The resulting LSD profiles feature a central wavelength of $625 \mathrm{~nm}$, an effective Landé factor of 1.25 and a mean relative depth with respect to the continuum of 0.686 . Zeeman signatures of full-amplitude up to $0.3 \%$ of the unpolarized continuum level are most of the time detected in the Stokes $V$ spectra. We observe a modulation of the Zeeman signatures, with a sign switch occurring at mid-time throughout the run. In order to ensure that the LSD profiles are not affected by the high level of noise in the bluest part of the spectra ( $\mathrm{S} / \mathrm{N}$ typically of 10-20 for the orders centered on wavelengths lower than $400 \mathrm{~nm}$ ), we also extracted the LSD profiles using only the mask lines redder than $400 \mathrm{~nm}$. As the resulting line profiles and magnetic analysis were marginally 


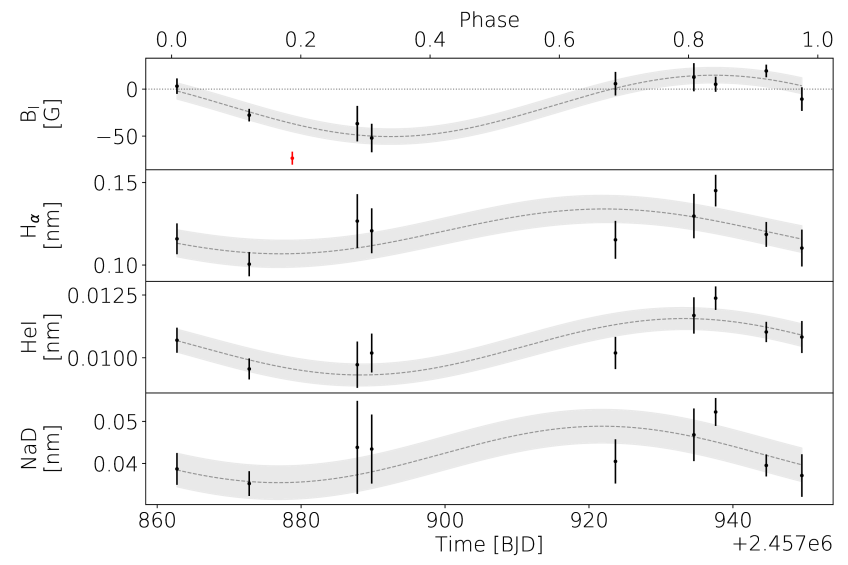

Figure 1. From top to bottom: time-series of the longitudinal field $\mathrm{B}_{\ell}$, and $\mathrm{H} \alpha \mathrm{He}$ I and $\mathrm{Na}$ D equivalent widths. In each panel, we show the data points as black dots (with $\pm 1 \sigma$ error bars), and a simple sine-wave linearly fitted to the data points using a least-squares estimator assuming $P_{\text {rot }}=89.8 \mathrm{~d}$ in gray dashed lines. Error bars from photon and detector noise on the equivalent widths of the activity proxies (3 lower panels) were respectively scaled up by a factor of about 100,5 and 10 to tentatively account for their intrinsic variability. In each panel, the gray bands indicate the $\pm 1 \sigma$ error bands of the fit. In the top panel, the horizontal dotted line indicates the zero level. The chromospheric indices are $35 \%$ to $85 \%$ larger than the median values of the time-series at phase 0.187 , which is interpreted by a stellar flare occurring shortly before the observation. This measurement was not included in the sine-wave fit to the chromospheric indices and is not displayed here for clarity purposes. As a precautionary measure, we also discarded the $\mathrm{B}_{\ell}$ value at phase 0.187 in the fitting procedure, even though it only marginally impacts the results of the fit.

impacted by the process, we kept using the LSD profiles computed using the full atomic line mask.

\section{MAGNETIC ANALYSIS}

\subsection{Longitudinal field}

For each observation, we compute the line-of-sight projection of the magnetic field, $\mathrm{B}_{\ell}$, by integrating our Stokes $I$ and $V$ LSD profiles on a $23 \mathrm{~km} \mathrm{~s}^{-1}$-wide window ${ }^{2}$ using the method detailed in Donati et al. (1997). $\mathrm{B}_{\ell}$ is a simple proxy of the field geometry, and its temporal fluctuations are known to provide reliable information on the stellar rotation period, $P_{\text {rot }}$ (Donati et al. 2006c; Hébrard et al. 2016). The longitudinal field values, are given in Table 1 and range typically from -73 to $20 \mathrm{G}$ with a median $1 \sigma$-uncertainty of $10 \mathrm{G}$. We model the $\mathrm{B}_{\ell}$ time-series using a simple sine-wave and use $\chi^{2}$ statistics to estimate $P_{\text {rot }}$. We find that the $\mathrm{B}_{\ell}$ time-series is modulated at $P_{\text {rot }}=90 \pm 6 \mathrm{~d}$. The best fit to the $\mathrm{B}_{\ell}$ time-series is shown in the top panel of Fig. 1 (reaching a reduced- $\chi^{2}$, denoted hereafter $\chi_{\mathrm{r}}^{2}$, of 1.0).

\subsection{Magnetic reconstruction of Proxima Cen}

We use ZDI (Semel 1989; Brown et al. 1991; Donati \& Brown $1997)$ to invert the Stokes $V$ LSD profiles into a map of the large-

2 The integration window is chosen to include the full width of the observed circularly-polarized Zeeman signatures (see the left panel of Fig. 2).

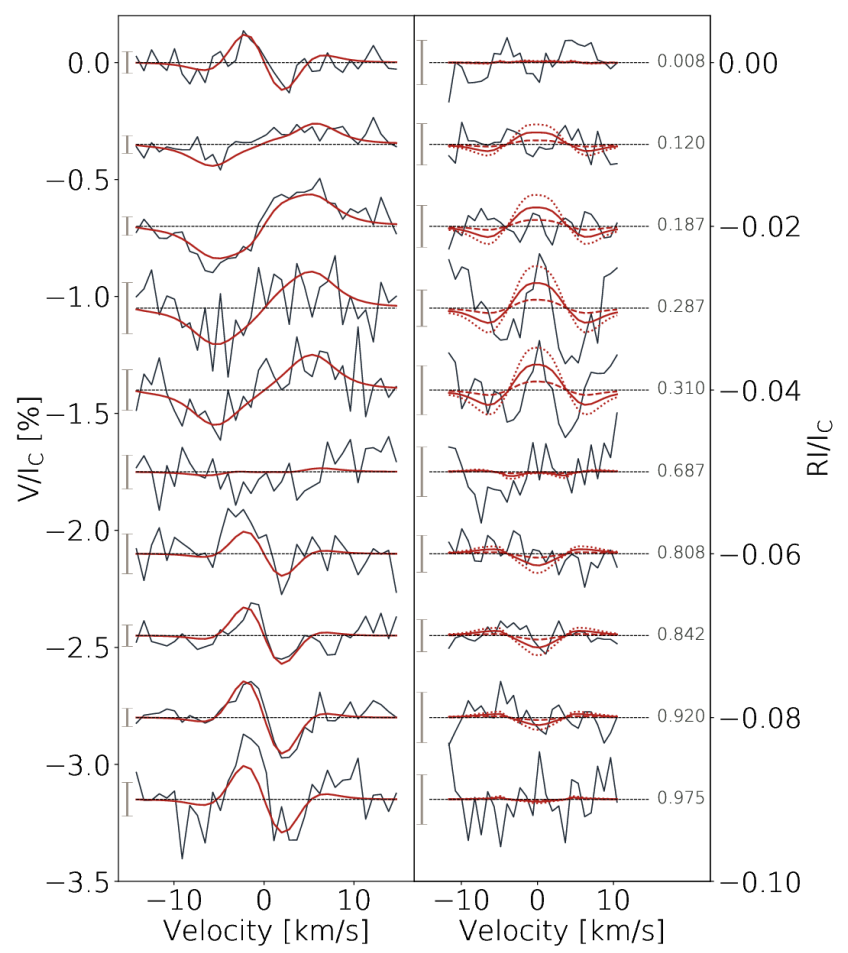

Figure 2. Left panel: Time-series of the observed circularly-polarized Zeeman signatures of Proxima Cen (black lines) and maximum-entropy reconstruction (red lines) in the stellar rest frame. Right panel: Median-subtracted Stokes $I$ LSD profiles (RI; black solid lines) and predictions using the magnetic topology presented in Sec. 3.2, assuming $f_{\mathrm{I}} / f_{\mathrm{V}}$ of 1 (red dashed lines), 3 (red solid lines) and 5 (red dotted lines, see the text for a definition of $f_{\mathrm{V}}$ and $f_{\mathrm{I}}$ ). The $\pm 1 \sigma$ error bars on each LSD line are indicated on the left side of each profile in both panels, while the corresponding stellar rotation phase, defined assuming $P_{\text {rot }}=89.8 \mathrm{~d}$ and the initial date $\mathrm{BJD}=2457862.0$,

are written on the right side of the right panel.

scale magnetic field at the surface of Proxima Cen. ZDI exploits the rotational modulation of Zeeman signatures to retrieve the poloidal and toroidal components of the large-scale magnetic field, both expressed as weighted sums of spherical harmonics (Donati et al. 2006c). For a given magnetic map, the stellar surface is sampled into a dense grid of $\sim 10000$ cells, for each of which ZDI computes local Stokes $I$ and $V$ profiles using analytical expressions from the Unno-Rachkovski's solution of the radiative transfer equation, assuming a plane-parallel Milne-Eddington atmosphere (Unno 1956). The width and depth of the local profiles are chosen in a way to minimize the $\chi_{\mathrm{r}}^{2}$ between the synthetic intrinsic profile and the median observed one. The local profiles are Doppler-shifted to the projected rotational velocity and weighted depending on limb darkening (assuming a linear law of coefficient 0.8; Claret et al. 2012), stellar inclination and local brightness, before being combined together into global Stokes $I$ and $V$ profiles. The time-series of the synthetic profiles are iteratively compared to the observed LSD profiles until reaching the maximum entropy solution for a given level of $\chi_{\mathrm{r}}^{2}$.

Using ZDI, we perform magnetic reconstructions of the stellar surface at a given level of entropy for different values of the stellar rotation period $P_{\text {rot }}$. By fitting a paraboloid to the resulting distribution of $\chi^{2}$, we find $P_{\text {rot }}=89.8 \pm 4.0 \mathrm{~d}$, in good agreement with the value of the rotation period obtained from the $\mathrm{B}_{\ell}$ time-series (see Sec. 3.1). A similar process allows to find a stellar inclination of 

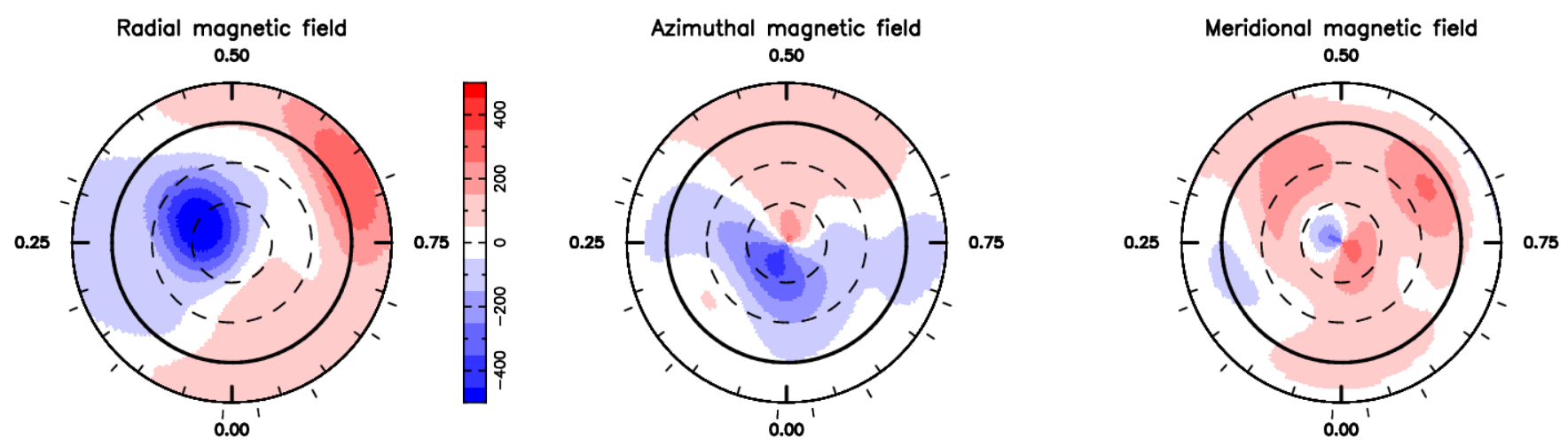

Figure 3. Surface distribution of the radial (left panel), azimuthal (middle panel), and meridional (right panel) components of the large-scale magnetic field of Proxima Cen. The star is described as a flattened polar view, where the circles indicate the equator (solid line) and $-30^{\circ}, 30^{\circ}$, and $60^{\circ}$ parallels (dashed lines). Ticks around the star mark the observations. Magnetic fields are expressed in G. Note that each cell features a filling factor of $f_{\mathrm{V}}=0.1$.

$i=47 \pm 7^{\circ}$ for Proxima Cen. In what follows, the phase of the star is defined assuming $P_{\text {rot }}=89.8 \mathrm{~d}$ and the initial date $\mathrm{BJD}=2457862$, corresponding to the beginning of our observations. From $P_{\text {rot }}, i$ and the stellar radius $R_{\mathrm{S}}$, we find a projected rotational velocity, $v \sin i$, of $0.06 \pm 0.01 \mathrm{~km} \mathrm{~s}^{-1}$ and use this value in the ZDI reconstruction. Given the low $v \sin i$ of Proxima Cen, we limit the spherical harmonic expansion to a degree $\ell=5$. Similarly to Morin et al. (2008), we introduce a filling factor $f_{\mathrm{V}}$ defined as the fraction of each cell (constant over the stellar surface) that contributes to polarized Zeeman signatures, yielding an integrated flux of the large-scale field equal to $B_{\mathrm{V}}$ over the cell. We find that $f_{\mathrm{V}}=0.1$ minimizes the $\chi_{\mathrm{r}}^{2}$ of the fit to both Stokes $V$ and Stokes $I$ LSD profiles (see Sec. 3.3).

The best fit to the observed Stokes $V$ LSD profiles is shown in the left panel of Fig. 2. We reach a $\chi_{\mathrm{r}}^{2}$ of 1.55 from an initial value of 3.7. The Stokes $V$ time series cannot be fit to a lower $\chi_{\mathrm{r}}^{2}$ level, possibly due to the intrinsic variability of the large-scale magnetic field over our observing run or to a slight under-estimation of the assumed error bars. The resulting magnetic topology is shown in Fig. 3. The average reconstructed large-scale magnetic field is $\left.<B_{\mathrm{V}}\right\rangle=B \times f_{\mathrm{V}}=200 \mathrm{G}$, meaning that $B$, the typical field strength present on $f_{\mathrm{V}}=10 \%$ of the stellar surface, is equal to $\sim 2 \mathrm{kG}$. The magnetic topology is mainly poloidal ( $92 \%$ of the magnetic energy budget), with a dominant dipolar contribution ( $60 \%$ of the poloidal energy) featuring a dipole of $135 \mathrm{G}$, tilted at $51^{\circ}$ to the rotation axis towards phase 0.28 . We also note a significant quadripolar component ( $22 \%$ of the poloidal energy), confirming the complex topology of the large-scale field. Moreover, we find a moderate fraction of magnetic energy in axisymmetric modes, $f_{\mathrm{axi}}=44 \%$. Finally, no information on the stellar differential rotation can be inferred from the Stokes $V$ spectra, as the total time-span of the observations barely covers a single stellar rotation.

\subsection{Zeeman broadening in unpolarized line profiles}

We observe a modulation of Zeeman broadening in the Stokes $I$ LSD profiles. In particular, the profiles appear significantly broadened around phase 0.3 , when the magnetic pole points towards the observer. We generate a time-series of synthetic Stokes I profiles from the magnetic topology obtained in Sec. 3.2. As for the modeling of Stokes $V$ spectra, we introduce a filling factor $f_{\mathrm{I}}$, also constant over the stellar surface and defined as the fractional area of each cell containing a small-scale magnetic field of strength $B_{\mathrm{I}}=B_{\mathrm{V}} / f_{\mathrm{V}}$, i.e., assumed to be distributed as the large-scale field $B_{\mathrm{V}}$ (see Morin et al. 2008 , for a more extensive definition of $f_{\mathrm{I}}$ ). The difference between $f_{\mathrm{I}}$ and $f_{\mathrm{V}}$ is explained by the fact that circular polarization signatures and Zeeman broadening probe different scales of the magnetic field. On small scales, regions with opposite magnetic polarities produce circularly-polarized signals that almost completely cancel out, which is not the case for the Zeeman broadening in Stokes $I$ spectra. We compare the Stokes $I$ LSD profiles to the synthetic ones assuming various values for $f_{\mathrm{I}}$ and find that $f_{\mathrm{I}} \sim 3 f_{\mathrm{V}}$ minimizes the $\chi^{2}$ of the residuals in the central regions of the Stokes $I$ profiles (i.e., within $\pm 6 \mathrm{~km} \mathrm{~s}^{-1}$ of the line center) where the modulation of the Zeeman broadening is largest (see the left panel of Fig. 2). We however caution that, being based on the simplifying approximation that $B_{\mathrm{I}}$ is distributed as $B_{\mathrm{V}}$, our measurement of $f_{\mathrm{I}} / f_{\mathrm{V}}$ is likely no more than a rough estimate. Using $f_{\mathrm{I}}=3 f_{\mathrm{V}}$, we find an unsigned magnetic flux density $\left\langle B_{\mathrm{I}}>f_{\mathrm{I}}=\left\langle B_{\mathrm{V}}>f_{\mathrm{I}} / f_{\mathrm{V}} \sim 600 \mathrm{G}\right.\right.$, consistent with the flux density measured from the Zeeman broadening of unsigned lines in Reiners \& Basri (2008). The ratio $f_{\mathrm{V}} / f_{\mathrm{I}} \sim 0.33$ is apparently higher than that typically measured for FC M dwarfs when this ratio was shown to reach 0.15 when the dynamo is saturated (Reiners \& Basri 2009; Morin et al. 2010; See et al. 2019). The reasonably good agreement between the large- and small-scale magnetic topologies and the high ratio $f_{\mathrm{V}} / f_{\mathrm{I}}$ suggest that the underlying dynamo process is apparently efficient at injecting the magnetic field into the largest spatial scales. Finally, assuming larger values for $f_{\mathrm{V}}$ in the magnetic reconstruction yields poorer fits to the modulation of the Zeeman broadening in the Stokes $I$ LSD profiles, confirming that $f_{\mathrm{V}}=0.1$ is a good estimate of the filling factor (with a typical uncertainty of $0.03)$.

\section{DISCUSSION AND CONCLUSION}

In this study, we report the first detection of a large scale magnetic field at the surface of Proxima Centauri. Ten spectropolarimetric observations, obtained with HARPS-Pol from April to July 2017, exhibit Zeeman signatures whose modulation indicates a stellar rotation period of $89.8 \pm 4.0 \mathrm{~d}$ and suggests a stellar inclination of $47 \pm 7^{\circ}$. We find a large-scale magnetic field of $\sim 200 \mathrm{G}$, whose topology is mainly poloidal, and features a dipole component of $135 \mathrm{G}$ tilted at $51^{\circ}$ to the rotation axis. Due to the low $v \sin i$ of the star, we mostly access the largest scales of the magnetic field (i.e., $\ell \leq 5$ ). Our resulting magnetic topology is thus expected to be no more 


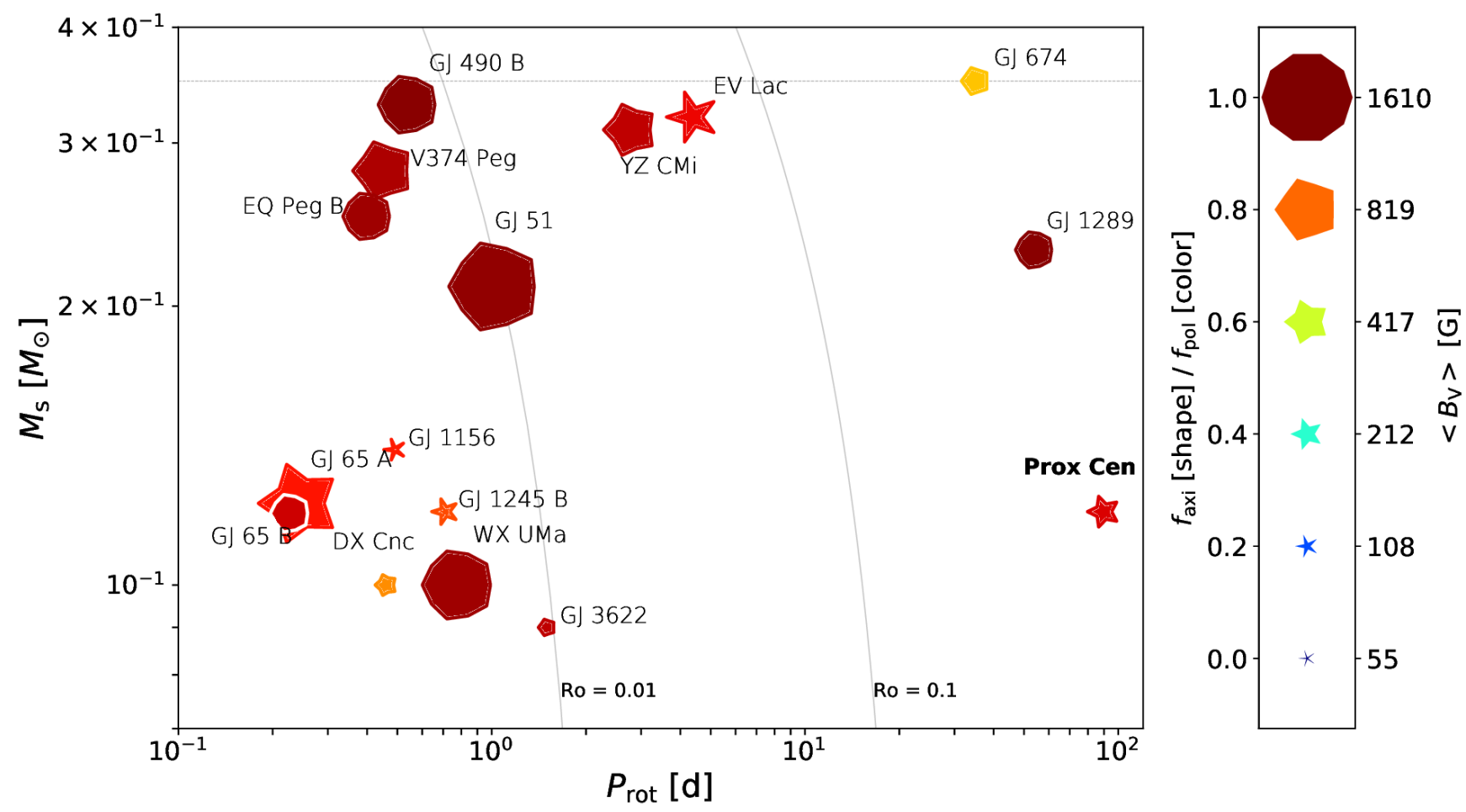

Figure 4. Rotation period-mass diagram of FC M dwarfs with reconstructed magnetic topology. The size of the symbols is proportional the strength of the magnetic field, their shapes represent their degree of axisymmetry (decagons and stars with $f_{\mathrm{axi}}$ of 1 and 0 respectively), and their colors refer to the fraction of poloidal field of the reconstruction (blue and red standing respectively for pure toroidal and poloidal fields). Contours of constant Rossby numbers of 0.1 and 0.01, computed using Wright et al. (2018) empirical relationship, are plotted in gray solid lines. The horizontal line indicate the theoretical full-convection limit $\left(\sim 0.35 \mathrm{M}_{\odot}\right.$ Baraffe et al. 1998). Except for Proxima Cen, the data originate from the magnetic analyses presented in Morin et al. (2008); Phan-Bao et al. (2009); Morin et al. (2010, 2011); Kochukhov \& Lavail (2017); Moutou et al. (2017).

than weakly affected by the gap in our observations between phases 0.310 and 0.687 . The circularly-polarized magnetic flux density is only $\sim 3 \times$ smaller than the flux measured from the Zeeman broadening of unsigned lines, which suggests that the underlying dynamo process is efficient at injecting magnetic energy in the largest spatial scales. As illustrated in the rotation period-mass diagram shown in Fig. 4, Proxima Cen's magnetic properties appear similar in term of field strength, fraction of poloidal energy, and degree of axisymmetry, to those of the group of FC M dwarfs with saturated dynamo exhibiting multipolar large-scale fields (Morin et al. 2010). However, Proxima Cen has the highest ratio $\left\langle B_{\mathrm{V}}\right\rangle \mid\left\langle B_{\mathrm{I}}\right\rangle$ reported so far, whereas this ratio is generally significantly weaker for stars in the multipolar regime $(\sim 6 \%)$ than for stars in the dipole dominated group $(\sim 15 \%)$. These results, if confirmed by new spectropolarimetric observations of Proxima Cen and of other slowly-rotating FC stars, challenge our understanding of dynamo generation in Mdwarfs. We now discuss the implications of the magnetic analysis on the chromospheric activity and magnetic cycle of the star, and on its extended magnetospheric structure.

\subsection{Magnetic activity and cycle of Proxima Cen}

Optical chromospheric activity indicators are known to be wellcoupled to bright and dark features in the case of the Sun. Comparing their temporal evolution with the magnetic topology recovered with ZDI provides us with valuable information on the magnetic connections between the photosphere and the chromosphere. We compute the equivalent width of $\mathrm{H} \alpha(6562.808 \AA$ ), He I D3 (5875.62 $\AA$ ), and Na I D1 and D2 (resp. 5895.92 and $5889.95 \AA$ ) chromospheric lines $^{3}$. The integration windows are those defined in Gomes da Silva et al. (2011) for late-type stars.

As shown in Fig. 1, the time-series of chromospheric activity indicators appear modulated at $P_{\text {rot }}$. The chromospheric emission in the $\mathrm{He}$ I line varies in phase with the magnetic field, reaching its minimum at phase $0.29 \pm 0.06$, when the negative pole comes closest to the observer (i.e., at phase $0.34 \pm 0.03$ ), and its maximum when the magnetic equator gets closer to the line-of-sight (phase $\sim 0.8$ ). The emissions in the cores of $\mathrm{H} \alpha$ and $\mathrm{Na} \mathrm{D}$ lines are shifted by $\sim 0.2$ in phase with respect to the magnetic field, with minimums of emission respectively reached at phases $0.17 \pm 0.07$ and $0.17 \pm 0.08$ (consistent at $2 \sigma$ with the phasing of the magnetic field). This shift is most likely attributable to (i) a complex distribution of the chromospheric material which might be differently probed in $\mathrm{He}$ I and $\mathrm{H} \alpha / \mathrm{Na} \mathrm{D}$ lines or (ii) to intrinsic stellar variability (e.g., flares or slow temporal evolution of the stellar chromosphere). The observed modulation of the chromospheric indices may reflect the coronal hole (associated with open field lines and presumably darkest at the chromospheric level) going in and out of view as the star rotates, and being best and least visible at phases 0.3 and 0.8 respectively.

3 Note that, due to the very low $\mathrm{S} / \mathrm{N}$ in the bluest parts of the spectra, we could not reliably estimate the equivalent width of $\mathrm{Ca} \mathrm{H} \& \mathrm{~K}$ lines from our observations. 
A full radiative transfer computation would be needed to confirm this suggestion.

Our spectropolarimetric observations take place near Proxima Cen's activity maximum (Suárez Mascareño et al. 2016; Wargelin et al. 2017). If, as suggested in the literature, Proxima Cen undergoes a 7 yr-solar-like activity cycle powered by a $\alpha \Omega$-dynamo process (e.g., Yadav et al. 2016), one would expect the magnetic field to oscillate from a nearly axisymmetric dipole, at the activity minimum, to a weaker less axisymmetric multipolar field, at activity maximum (Kitchatinov et al. 2014). In particular, the total fractions of axisymmetric and poloidal magnetic energies reconstructed by ZDI seem to be excellent proxies of the magnetic cycle for partly-convective stars (see Lehmann et al., 2020, in prep.). Hence the interest to keep monitoring Proxima Cen's magnetic properties throughout the activity cycle.

Another major interest of future spectropolarimetric observations is to constrain the stellar differential rotation and its evolution with the activity cycle. Our measurement of $P_{\text {rot }}$, although significantly higher than the average photometric rotation period (e.g., $83.10 \pm 0.05 \mathrm{~d}$ in Wargelin et al. 2017), is consistent with the period of $90.1 \mathrm{~d}$ measured by Wargelin et al. (2017) in 2009, i.e., around the previous activity maximum. The authors do not find any correlation between the photometric $P_{\text {rot }}$ and the cycle phase, which suggests that the photometric period is not so good proxy of the activity cycle for proxima Cen. On the other hand, the variation of $P_{\text {rot }}$ measured from circularly-polarized Zeeman signatures might be a good indicator of the magnetic cycle, as the large-scale magnetic field is directly linked to the underlying dynamo processes.

Finally, near-infrared spectroscopic observations of Proxima Cen with upcoming instruments like NIRPS (Bouchy et al. 2017; Wildi et al. 2017) will complement the analysis by allowing the measurement of other activity indicators (e.g., based on the emission flux in the core of the $\mathrm{He} I$ triplet at $1083 \mathrm{~nm}$, and Paschen $\beta$ ), and by constraining the ratio $\left\langle B_{\mathrm{I}}\right\rangle \mid\left\langle B_{\mathrm{V}}\right\rangle$ thanks to the larger Zeeman broadening in the near-infrared than in the optical domain.

\subsection{Extended magnetosphere and implications for Proxima-b}

Given that the corotation radius of proxima Cen is $\approx 285 R_{\mathrm{S}}$, field lines are not expected to break under the effect of centrifugal forces, but rather under that of the stellar wind. We estimate the typical radius of the spherical Alvèn surface, $R_{\mathrm{A}}$, above which field lines open under stellar wind ram pressure. We first compute the magnetic confinement parameter, $\eta$, defined in ud-Doula \& Owocki (2002) as the ratio between magnetic and wind kinetic energy densities, assuming a magnetic field of $135 \mathrm{G}$. Using a stellar mass-loss rate of $2 \times 10^{-15} \mathrm{M}_{\odot} / \mathrm{yr}$ and a stellar wind terminal velocity of $400 \mathrm{~km} \mathrm{~s}^{-1}$ (Wood et al. 2001), we obtain a confinement parameter as large as $\eta \sim 3.9 \times 10^{5}$. At large distance from the star (typically a few stellar radii), the extended magnetic field of Proxima Cen is mainly dipolar. Moreover, Proxima Cen features a corona likely reaching the MK level, which would be enough to entirely ionize the stellar wind (as demonstrated for a few M dwarfs of earlier types; e.g., Vidotto et al. 2019; Mesquita \& Vidotto 2020). Under these conditions, ud-Doula \& Owocki (2002) semi-analytical relations provide us with a first order estimate of the equatorial Aflvèn radius of $\sim 25 R_{\mathrm{S}}$. At this distance, the magnetic field of Proxima Cen is mostly dipolar, as evidenced by the extrapolation of the potential field lines shown at phase 0.8 in Fig. 5, computed using the field-extrapolation technique described in Jardine et al. (1999). Proxima-b, that orbits at a distance of $\sim 70 R_{\mathrm{S}}>R_{\mathrm{A}}$ from its host star, is thus expected to lie in the superAlfvenic regime, where no direct star-planet magnetic connection

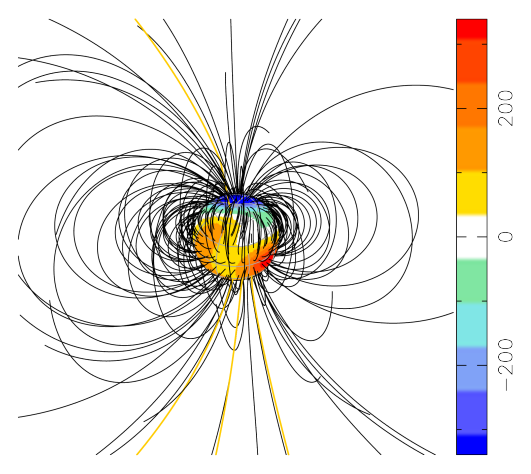

Figure 5. Potential field extrapolated from the reconstructed magnetic topology at phase 0.8 (around the maximum $\mathrm{H} \alpha$ emission). Open and close field lines are respectively shown in black and yellow solid lines. The color scale depicts the strength of the radial component of the large-scale magnetic field at the stellar surface. In order to better visualize the variations of the radial field at the surface of the star (and then more directly compare it with the potential field extrapolation), we show the star as seen from a distance of $5 R_{\mathrm{S}}$. The source surface at which the magnetic field lines are radial is set to $25 R_{\mathrm{S}}$ for this plot.

occurs (Strugarek et al. 2015). We however caution that magnetohydrodynamical simulations are needed to validate this conclusion (e.g., in a way similar to Strugarek et al. 2015; Vidotto \& Donati 2017; Folsom et al. 2018; Strugarek et al. 2019; Folsom et al. 2020). Finally, constraining the evolution of the size of the magnetosphere with the stellar activity cycle will help unveiling potential SPIs that may affect the magnetosphere of Proxima-b.

As discussed in Vidotto et al. (2014), the value $f_{\text {axi }}$ of the ZDI reconstruction correlates with the flux of galactic cosmic rays received by the planet (based on solar system observations studied in Wang et al. 2006). The moderate value of $f_{\text {axi }}$ for Proxima Cen indicates that, its close-in planet is relatively well-shielded from galactic cosmic rays at the time of the observations, which has implications for habitability condition studies (Rimmer \& Helling 2013; Sadovski et al. 2018). Our analysis confirms the strength of the large-scale field assumed in Ribas et al. (2016), which implies that Proxima $b$ could likely sustain a magnetosphere of $\sim 2-3$ planetary radii under a wind terminal velocity similar to that assumed to compute the Alfvèn radius of the star. Moreover, extended non-axisymmetric magnetic fields tend to favour axisymmetric distributions of the wind mass flux (Vidotto et al. 2014). This suggests that the size of Proxima-b's magnetosphere undergoes little variations at the time of observations, contrary to what was predicted by recent studies based on a different magnetic topology (e.g., Garraffo et al. 2016, who assume a $\mathrm{Gj}$ 51-like magnetic geometry whose maximum strength is rescaled to $600 \mathrm{G}$ ).

More generally, our study confirms that the large-scale magnetic topology of slowly-rotating FC M dwarfs can be retrieved with ZDI. Our results open up promising prospects for the study of the magnetic field of FC M dwarfs and their interactions with closein planets. In particular, new generation near-infrared spectropolarimeters like SPIRou at the Canada-France-Hawaii Telescope (Donati et al. 2018) and its upcoming twin, SPIP, at the Pic du 
Midi Observatory, may bring decisive constraints on the dynamo processes operating at the lower end of the main sequence.

\section{ACKNOWLEDGEMENTS}

This project was funded by the European Research Council (ERC) under the $\mathrm{H} 2020$ research \& innovation programme (grant agreements \#740651 NewWorlds). This work is based on observations made with ESO Telescopes at the La Silla Paranal Observatory. We thank the referee for valuable comments and suggestions which helped us improving an earlier version of the manuscript.

\section{DATA AVAILABILITY}

This paper includes data collected by the HARPS-Pol spectropolarimeter, which is publicly available from the ESO Science Archive Facility (program ID: 099.C-0334).

\section{REFERENCES}

Anglada-Escudé G., et al., 2016, Nature, 536, 437

Astudillo-Defru N., Delfosse X., Bonfils X., Forveille T., Lovis C., Rameau J., 2017a, A\&A, 600, A13

Astudillo-Defru N., et al., 2017b, A\&A, 602, A88

Baraffe I., Chabrier G., Allard F., Hauschildt P. H., 1998, A\&A, 337, 403

Barnes R., et al., 2016, arXiv e-prints, p. arXiv:1608.06919

Berta-Thompson Z. K., et al., 2015, Nature, 527, 204

Bessell M. S., 1991, AJ, 101, 662

Bouchy F., et al., 2017, The Messenger, 169, 21

Brown S. F., Donati J. F., Rees D. E., Semel M., 1991, A\&A, 250, 463

Claret A., Hauschildt P. H., Witte S., 2012, A\&A, 546, A14

Dittmann J. A., et al., 2017, Nature, 544, 333

Donati J. F., Brown S. F., 1997, A\&A, 326, 1135

Donati J. F., Landstreet J. D., 2009, ARA\&A, 47, 333

Donati J. F., Semel M., Carter B. D., Rees D. E., Collier Cameron A., 1997, MNRAS, 291, 658

Donati J.-F., Forveille T., Collier Cameron A., Barnes J. R., Delfosse X., Jardine M. M., Valenti J. A., 2006a, Science, 311, 633

Donati J. F., Catala C., Landstreet J. D., Petit P., 2006b, in Casini R., Lites B. W., eds, Astronomical Society of the Pacific Conference Series Vol. 358, Solar Polarization 4. p. 362

Donati J. F., et al., 2006c, MNRAS, 370, 629

Donati J.-F., et al., 2018, SPIRou: A NIR Spectropolarimeter/High-Precision Velocimeter for the CFHT. p. 107, doi:10.1007/978-3-319-553337_107

Folsom C. P., et al., 2018, MNRAS, 481, 5286

Folsom C. P., Fionnagáin D. Ó., Fossati L., Vidotto A. A., Moutou C., Petit P., Dragomir D., Donati J. F., 2020, A\&A, 633, A48

France K., et al., 2016, ApJ, 820, 89

Gaia Collaboration et al., 2018, A\&A, 616, A1

Garcia-Sage K., Glocer A., Drake J. J., Gronoff G., Cohen O., 2017, ApJ, 844, L13

Garraffo C., Drake J. J., Cohen O., 2016, ApJ, 833, L4

Gastine T., Duarte L., Wicht J., 2012, A\&A, 546, A19

Gastine T., Morin J., Duarte L., Reiners A., Christensen U. R., Wicht J., 2013, A\&A, 549, L5

Gillon M., et al., 2017, Nature, 542, 456

Gomes da Silva J., Santos N. C., Bonfils X., Delfosse X., Forveille T., Udry S., 2011, A\&A, 534, A30

Hébrard É. M., Donati J. F., Delfosse X., Morin J., Moutou C., Boisse I., 2016, MNRAS, 461, 1465

Jardine M., Barnes J. R., Donati J.-F., Collier Cameron A., 1999, MNRAS, 305, L35

Kasting J. F., Whitmire D. P., Reynolds R. T., 1993, Icarus, 101, 108
Kiraga M., Stepien K., 2007, Acta Astron., 57, 149

Kitchatinov L. L., Moss D., Sokoloff D., 2014, MNRAS, 442, L1

Kochukhov O., Lavail A., 2017, ApJ, 835, L4

Kurucz R. L., 1993, SYNTHE spectrum synthesis programs and line data Lammer H., et al., 2007, Astrobiology, 7, 185

Mayor M., et al., 2003, The Messenger, 114, 20

Meadows V. S., et al., 2018, Astrobiology, 18, 133

Mesquita A. L., Vidotto A. A., 2020, MNRAS, 494, 1297

Morin J., et al., 2008, MNRAS, 390, 567

Morin J., Donati J. F., Petit P., Delfosse X., Forveille T., Jardine M. M., 2010, MNRAS, 407, 2269

Morin J., Dormy E., Schrinner M., Donati J. F., 2011, MNRAS, 418, L133

Moutou C., et al., 2017, MNRAS, 472, 4563

Passegger V. M., Wende-von Berg S., Reiners A., 2016, A\&A, 587, A19

Phan-Bao N., Lim J., Donati J.-F., Johns-Krull C. M., Martín E. L., 2009, ApJ, 704, 1721

Pizzolato N., Maggio A., Micela G., Sciortino S., Ventura P., 2003, A\&A, 397,147

Reiners A., Basri G., 2008, A\&A, 489, L45

Reiners A., Basri G., 2009, A\&A, 496, 787

Ribas I., et al., 2016, A\&A, 596, A111

Ribas I., Gregg M. D., Boyajian T. S., Bolmont E., 2017, A\&A, 603, A58

Rimmer P. B., Helling C., 2013, ApJ, 774, 108

Sadovski A. M., Struminsky A. B., Belov A., 2018, Astronomy Letters, 44, 324

See V., et al., 2019, ApJ, 876, 118

Semel M., 1989, A\&A, 225, 456

Shulyak D., Reiners A., Engeln A., Malo L., Yadav R., Morin J., Kochukhov O., 2017, Nature Astronomy, 1, 0184

Snik F., et al., 2011, The HARPS Polarimeter. p. 237

Strugarek A., Brun A. S., Matt S. P., Réville V., 2015, ApJ, 815, 111

Strugarek A., Brun A. S., Donati J. F., Moutou C., Réville V., 2019, ApJ, 881,136

Suárez Mascareño A., Rebolo R., González Hernández J. I., 2016, A\&A, 595, A12

Turbet M., Leconte J., Selsis F., Bolmont E., Forget F., Ribas I., Raymond S. N., Anglada-Escudé G., 2016, A\&A, 596, A112

Unno W., 1956, PASJ, 8, 108

Vidotto A. A., Donati J. F., 2017, A\&A, 602, A39

Vidotto A. A., Jardine M., Morin J., Donati J. F., Opher M., Gombosi T. I., 2014, MNRAS, 438, 1162

Vidotto A. A., Feeney N., Groh J. H., 2019, MNRAS, 488, 633

Wang Y. M., Sheeley N. R. J., Rouillard A. P., 2006, ApJ, 644, 638

Wargelin B. J., Saar S. H., Pojmański G., Drake J. J., Kashyap V. L., 2017, MNRAS, 464, 3281

West A. A., et al., 2011, AJ, 141, 97

Wildi F., et al., 2017, in Society of Photo-Optical Instrumentation Engineers (SPIE) Conference Series. p. 1040018, doi:10.1117/12.2275660

Wood B. E., Linsky J. L., Müller H.-R., Zank G. P., 2001, ApJ, 547, L49

Wright N. J., Newton E. R., Williams P. K. G., Drake J. J., Yadav R. K., 2018, MNRAS, 479, 2351

Yadav R. K., Christensen U. R., Morin J., Gastine T., Reiners A., Poppenhaeger K., Wolk S. J., 2015, ApJ, 813, L31

Yadav R. K., Christensen U. R., Wolk S. J., Poppenhaeger K., 2016, ApJ, 833, L28

ud-Doula A., Owocki S. P., 2002, ApJ, 576, 413

This paper has been typeset from a $\mathrm{T}_{\mathrm{E}} \mathrm{X} / \mathrm{LAT}_{\mathrm{E}} \mathrm{X}$ file prepared by the author. 